\title{
Modeling of Dynamic Hysteresis Based on Takagi-Sugeno Fuzzy Duhem Model
}

Sang-Yun Lee ${ }^{1}$, Mignon Park ${ }^{1}$, and Jaeho Baek ${ }^{2}$

${ }^{1}$ School of Electrical and Electronic Engineering, Yonsei University, Seoul, Korea

${ }^{2}$ Advanced R\&D Team (Digital Appliances), Samsung Electronics Co. Ltd., Suwon, Korea

\section{ljfis}

Received: Nov. 21, 2013

Revised : Dec. 23, 2013

Accepted: Dec. 25, 2013

Correspondence to: Jaeho Baek (jhoya97@gmail.com)

(CThe Korean Institute of Intelligent Systems

(c) This is an Open Access article distributed under the terms of the Creative Commons Attribution Non-Commercial License (http://creativecommons.org/licenses/ by-nc/3.0// which permits unrestricted noncommercial use, distribution, and reproduction in any medium, provided the original work is properly cited.

\begin{abstract}
In this study, we propose a novel method for modeling dynamic hysteresis. Hysteresis is a widespread phenomenon that is observed in many physical systems. Many different models have been developed for representing a hysteretic system. Among them, the Duhem model is a classical nonlinear dynamic hysteresis model satisfying the properties of hysteresis. The purpose of this work is to develop a novel method that expresses the local dynamics of the Duhem model by a linear system model. Our approach utilizes a certain type of fuzzy system that is based on Takagi-Sugeno (T-S) fuzzy models. The proposed T-S fuzzy Duhem model is achieved by fuzzy blending of the linear system model. A simulated example applied to shape memory alloy actuators, which have typical hysteretic properties, illustrates the applicability of our proposed scheme.
\end{abstract}

Keywords: Hysteresis, Modeling, Duhem model, Takagi-Sugeno fuzzy, Shape memory alloy actuator

\section{Introduction}

Hysteresis is a phenomenon that has long been observed in a wide variety of physical systems, including mechanical hysteresis, magnetic hysteresis, and material hysteresis. For instance, hysteresis displayed by shape memory alloy (SMA) during the process of a phase transition is an example of hysteresis in a mechanical system [1]. There is no precise agreement in the literature regarding the definition of hysteresis. In this paper, we follow the definition proposed by Mayergoyz [2]. According to this definition, a system is said to be hysteretic if its input-output relationship is a multi-branch nonlinearity. Figure 1 shows a typical hysteresis nonlinearity.

Modeling of hysteresis nonlinearity is an important goal of research in the field of hysteresis. For several decades, various models for hysteresis have been developed, such as the Preisach model [3] and Duhem model [4]. The Duhem model is a classical nonlinear dynamic model, whereas the Preisach model is not a dynamic model. Additionally, the Duhem model satisfies the properties of hysteresis, such as memory and rate-independence. Moreover, the Duhem model is able to tune the minor loop shapes independently from the major loop shapes. This increases the usefulness of the Duhem model in describing complicated minor loops. Hence, this model has been widely used to describe hysteretic systems, and many other models of hysteresis are related to the Duhem model [5]. For example, the Bouc-Wen model [6, 7] as well as the Coleman-Hodgdon model [8] are special cases of the Duhem model. The Chua- 


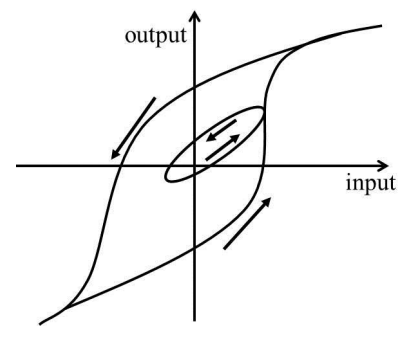

Figure 1. Hysteresis nonlinearity.

Stromsmoe model [9] is a generalization of the Duhem model

The hysteresis phenomenon is a typical nonlinear system. Hence, it is impossible to apply various linear system theories, and it is difficult to analyze a hysteresis system, even though the Duhem model expresses the dynamics of hysteresis well. To solve this problem, we propose a dynamic hysteresis model based on a Takagi-Sugeno (T-S) fuzzy model to express the local dynamics of a Duhem model with a linear system model. The proposed T-S fuzzy Duhem model is achieved by fuzzy blending of the linear system model. By using the proposed model, we can apply various linear system theories to a dynamic hysteresis model.

The remainder of this paper is organized as follows. In Section 2, we explain hysteresis and its properties. In Section 3 , the dynamics of the Duhem model are introduced. Section 4 presents the proposed T-S fuzzy Duhem model. Simulated results verifying the applicability of the proposed model are provided in Section 5. Concluding remarks are presented in the final section.

\section{Hysteresis and Its Properties}

\subsection{Hysteresis}

Hysteresis arises in diverse physical systems, including mechanical hysteresis, magnetic hysteresis, and material hysteresis. The word "hysteresis" has been derived from the Greek word "hysterein," which connotes lag, and hysteretic systems are generally described as having memory. Although there is no precise definition of hysteresis, our work follows the definition proposed by Mayergoyz. According to Mayergoyz, a system is said to be hysteretic if its input-output relationship is a multi-branch nonlinearity for which transitions from a branch to another branch occur at input extrema.

\subsection{Properties of Hysteresis}

Hysteresis has several properties. These properties are re-

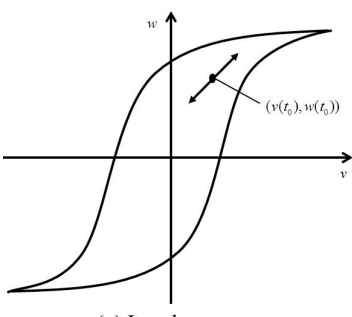

(a) Local memory

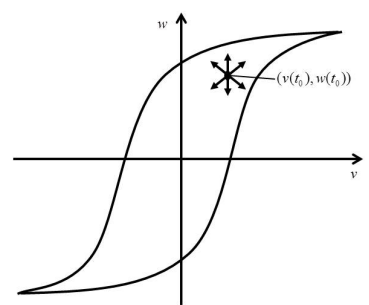

(b) Nonlocal memory
Figure 2. Hysteretic memory.

garded as inherent to hysteresis or as necessary conditions for a system to display hysteresis. Two of the most important properties of hysteresis are memory and rate-independence.

Mayergoyz has said that hysteresis can be regarded as nonlinearity with memory. Memory implies that the output may depend not only on the input but also on the previous evolution of the input. According to Mayergoyz, all hysteresis nonlinearities can be classified into two categories: hysteresis nonlinearities with local memories and hysteresis nonlinearities with nonlocal memories. For the hysteresis nonlinearities with local memories, the future output depends uniquely upon the future input for any given output. Figure 2(a) shows that the graph can follow only one path if the input increases and only one path if the input decreases. However, for the hysteresis nonlinearities with nonlocal memories, the future output depends not only upon the current output and future input but also on the past history of input extremum values. Hence, the graph can trace any number of paths, depending on the previous evolution of input, as shown in Figure 2(b).

The decisive characteristic differentiating hysteretic nonlinearities from all other systems with memory is the rateindependence property. Hysteresis is said to be rate-independent if the rate of change of input has no influence on branching, while variation in the branches of the hysteresis nonlinearity are determined only by the past extremum of the input. These properties often determine whether a hysteresis model is suitable for representing a system or not [5].

\section{Classical Duhem Model}

Many different mathematical models have been developed to express hysteresis nonlinearity, such as the Preisach model, Duhem model, Bouc-Wen model, and Chua-Stromsmoe model. Considering the usefulness of the dynamics provided by models and the capability of satisfying the above-mentioned properties, we chose a classical Duhem model to design the hysteresis 
model based on T-S fuzzy models.

The Duhem model is a differential-equation-based hysteresis model. For any differentiable hysteresis input $v(t)$ and any initial value $w_{0}$ of the hysteresis output, the hysteresis output $w$ is defined to be the solution of the following initial value problem:

$$
\begin{aligned}
\dot{w}(t) & =g_{+}(v(t), w(t))(\dot{v}(t))^{+}-g_{-}(v(t), w(t))(\dot{v}(t))^{-} \\
w(0) & =w_{0}
\end{aligned}
$$

The slopes of the hysteresis curves can be determined by slope functions $g_{+}$and $g_{-}$. The subscripts + and - represent increasing and decreasing curves, respectively. For $(\dot{v}(t))^{ \pm}$, the slope can be defined as

$$
(\dot{v}(t))^{ \pm}=\frac{|\dot{v}(t)| \pm \dot{v}(t)}{2} .
$$

By using the Duhem model with the appropriate slope functions for both increasing and decreasing conditions, we can model the hysteresis. Consequently, how to choose suitable slope functions is key for successful modeling using the Duhem model. The literature [1] proposes the use of Gaussian probability density functions (PDFs) as slope functions for the Duhem model, which is given by

$$
g_{ \pm}(v)=\frac{k}{\sigma_{ \pm} \sqrt{2 \pi}} \exp \left(-\frac{\left(v-\mu_{ \pm}\right)^{2}}{2 \sigma_{ \pm}^{2}}\right)
$$

where $v$ is the input, $k$ is the constant parameter, $\sigma^{2}$ is the variance, and $\mu$ is the mean of the Gaussian PDF. We assume that hysteresis curves have zero slopes at both the beginning and end of each curve. Hence, we can also choose Gaussian PDFs for the slope functions of the Duhem model. From Eqs. (1-3), we can observe that the major loop slope function is given by the differential equation

$$
\frac{d w}{d v}= \begin{cases}\frac{k}{\sigma_{+} \sqrt{2 \pi}} \exp \left(-\frac{\left(v-\mu_{+}\right)^{2}}{2 \sigma_{+}^{2}}\right), & \dot{v} \geq 0 \\ \frac{k}{\sigma_{-} \sqrt{2 \pi}} \exp \left(-\frac{\left(v-\mu_{-}\right)^{2}}{2 \sigma_{-}^{2}}\right), & \dot{v}<0 .\end{cases}
$$

An approach for representing the differential model of a minor loop was proposed by Likhachev. According to Likhachev, the major loop slope functions are multiplied by a scaling constant to obtain the minor loop slope functions. Consequently, the complete hysteresis model including both the major loop

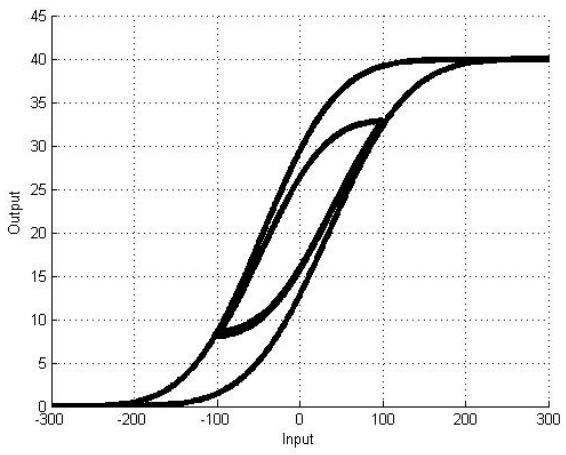

Figure 3. Hysteresis curves based on the Duhem model.

and the minor loop can be expressed as

$$
\frac{d w}{d v}= \begin{cases}k \frac{h_{-}(v)-w}{h_{-}(v)-h_{+}(v)} g_{+}(v), & \dot{v} \geq 0 \\ k \frac{w-h_{+}(v)}{h_{-}(v)-h_{+}(v)} g_{-}(v), & \dot{v}<0\end{cases}
$$

where

$$
h_{ \pm}(v)=\frac{1}{2}\left[1+\operatorname{erf}\left(\frac{v-\mu_{ \pm}}{\sigma_{ \pm} \sqrt{2}}\right)\right] .
$$

Figure 3 shows an example of hysteresis curves modeled by the Duhem model with Gaussian PDF slope functions. These curves consist of a major loop and a minor loop.

\section{T-S fuzzy Duhem Model}

In Eqs. (5) and (6), the Duhem model is expressed in the form of differential dynamic equations. Because these dynamics consist of nonlinear functions, various linear system theories and analysis techniques cannot be applied to the hysteresis system. To solve this problem, we propose a T-S fuzzy Duhem model, which expresses the local dynamics of the Duhem model by a linear system model. As expressed in [10], the $i$ rules of the T-S fuzzy models are of the following form:

$$
\begin{aligned}
& \text { If } z_{1}(t) \text { is } M_{i 1} \text { and } \cdots \text { and } z_{p}(t) \text { is } M_{i p} \\
& \begin{aligned}
\dot{x}(t) & =A_{i} x(t)+B_{i} u(t), \\
y(t) & =C_{i} x(t), \quad i=1,2, \ldots, r .
\end{aligned}
\end{aligned}
$$

Here, $M_{i j}$ is the fuzzy set, and $r$ is the number of model rules: $x(t) \in \Re^{n}$ is the state vector, $u(t) \in \Re^{m}$ is the input vector, $y(t) \in \Re^{q}$ is the output vector, and $A_{i} \in \Re^{n \times n}, B_{i} \in \Re^{n \times m}$, and $C_{i} \in \Re^{q \times n} ; z_{1}(t), \ldots, z_{p}(t)$ are known premise variables that may be functions of the state variables and time.

By modifying the differential equation of the Duhem model, we can apply a T-S fuzzy model to the Duhem model. Eq. (5) 
can be modified as

$$
\dot{w}=\left\{\begin{array}{cc}
-k \frac{g_{+}(v) \dot{v}}{h_{-}(v)-h_{+}(v)} w+k \frac{h_{-}(v) g_{+}(v) \dot{v}}{h_{-}(v)-h_{+}(v)}, & \dot{v} \geq 0 \\
k \frac{g_{-}(v) \dot{v}}{h_{-}(v)-h_{+}(v)} w-k \frac{h_{+}(v) g_{-}(v) \dot{v}}{h_{-}(v)-h_{+}(v)}, & \dot{v}<0 .
\end{array}\right.
$$

We define $v$ and $\dot{v}$ as premise variables $z_{1}(t)$ and $z_{2}(t)$, respectively. Then, the $i$ rules of the proposed T-S fuzzy Duhem model are derived as

$$
\begin{aligned}
& \text { If } v(t) \text { is } M_{i 1} \text { and } \dot{v}(t) \text { is } M_{i 2} \\
& \text { Then } \begin{aligned}
\dot{x}(t) & =A_{i} x(t)+B_{i}, \\
y(t) & =C_{i} x(t), \quad i=1,2, \ldots, r .
\end{aligned}
\end{aligned}
$$

where $x(t)=w(t)$ and $C_{i}=1$.

There are two approaches for constructing a fuzzy model. The identification approach, which uses input-output data, is suitable for plants that are too difficult to be expressed as analytical models. On the other hand, when a nonlinear dynamic model is obtainable, a fuzzy model can be modeled by a derivation from the given nonlinear system equations. Indeed, if we know the nominal values of the parameters $\mu_{ \pm}$and $\sigma_{ \pm}$, then we can use the second approach. In general, however, choosing the parameter values of the slope functions may be difficult. In such cases, the identification approach is more appropriate.

By using the proposed T-S fuzzy Duhem model, we can express the hysteretic system with a sublinear dynamic system. In fact, it is proven that T-S fuzzy models are universal approximators and used for various application in [11-15].

\section{Simulations}

This section presents a simulation of the T-S fuzzy Duhem model with models of SMA actuators. SMAs are metallic alloys that display the shape memory effect. When SMAs are cooled or heated to a different temperature, phase transitions can occurred. During the process of the phase transition, SMAs exhibit a hysteretic transformation. To demonstrate the effectiveness of the proposed method, we compare the simulation results for modeling using the Duhem model with those using the T-S fuzzy Duhem model. Simulations for two cases were executed to verify the applicability of the T-S fuzzy Duhem model. The first case is that the hysteresis curve displays only a major loop. The second case is that the hysteresis curve displays both a major loop and minor loop. Figures 4 and 5 show the temperature input signal used in the simulations. The range of the input is $\left[\begin{array}{ll}-300 & 300\end{array}\right]$. Table 1 shows the parameter values used in this modeling simulation. These parameters are

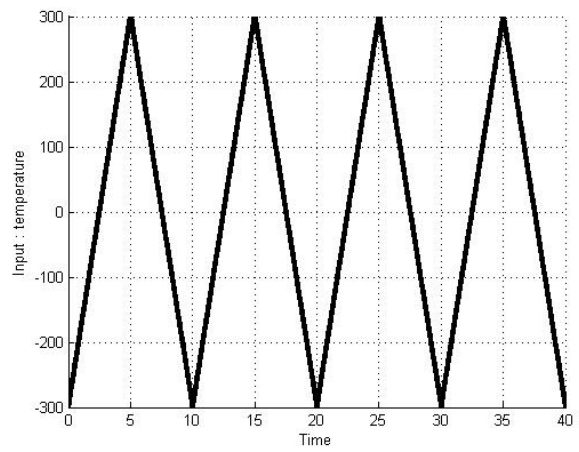

Figure 4. Input signal for major loop.

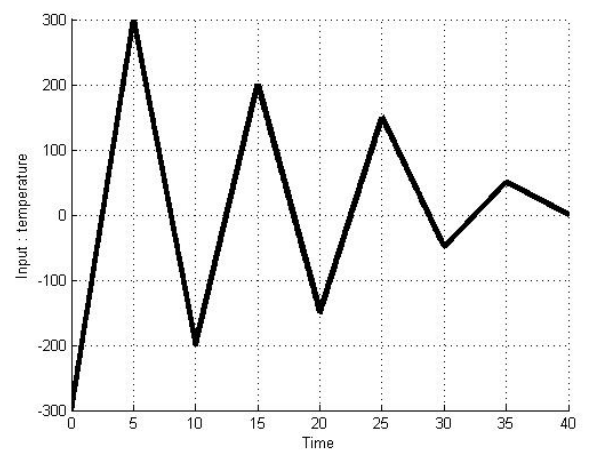

Figure 5. Input signal for mixed loop.

obtained from the literature [16].

As mentioned above, we can model the hysteresis of an SMA actuator by using the classical Duhem model. Under the above parameters and equations, hysteresis curves modeled by the classical Duhem model are shown in Figures 6 and 7 . Because an identification approach is chosen to construct T-S fuzzy systems, input-output pairs should be obtained. We can obtain input-output pairs from the simulation results for the classical Duhem model and construct a T-S fuzzy system with the data. Eleven fuzzy sets are defined as fuzzy sets of the first input $v$, as shown in Figure 8. To reflect two conditions of Eq. (9), two fuzzy sets are defined as fuzzy sets of the second input $\dot{v}$, as shown in Figure 9. These fuzzy sets reflect whether the SMA actuator is in a cooling state or heating state. By using the input-output pairs and the previous membership functions, we can determine the values of $A_{i}$ and $B_{i}$ in Eq. (11) for each rule. Consequently, we can plot the hysteresis curves modeled by the T-S fuzzy Duhem model, as in Figures 10 and 11

The results in Figures 10 and 11 exhibit the hysteresis nonlinearity well. More analytically, we computed modeling errors between the results for the classical Duhem model and the T-S fuzzy Duhem model. The modeling errors are shown in Fig- 
Table 1. Simulation parameters

\begin{tabular}{ccccc}
\hline$k$ & $\mu_{+}$ & $\mu_{-}$ & $\sigma_{+}$ & $\sigma_{-}$ \\
\hline 40 & 75 & 70 & 35 & -45 \\
\hline
\end{tabular}

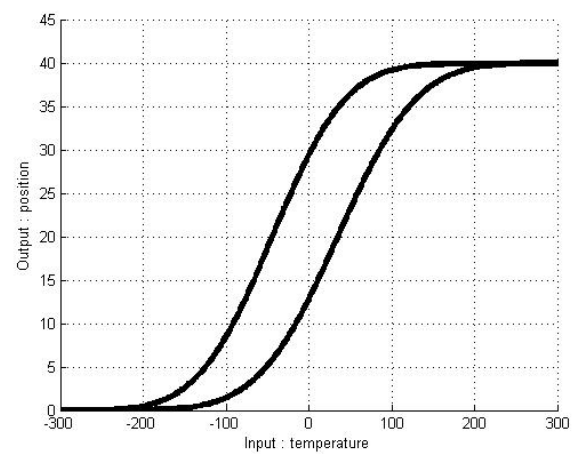

Figure 6. Hysteresis curves for the Duhem model with major loop.

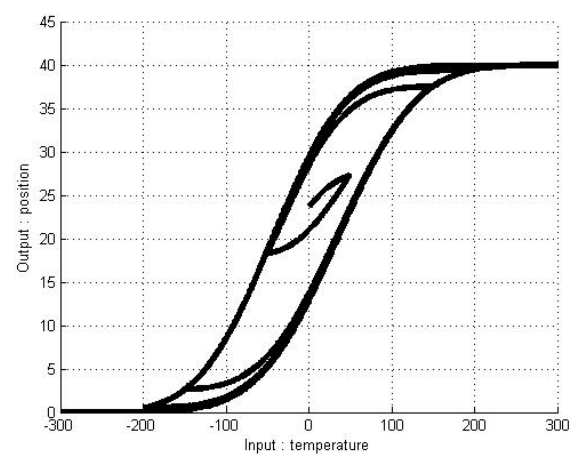

Figure 7. Hysteresis curves for the Duhem model with mixed loop.

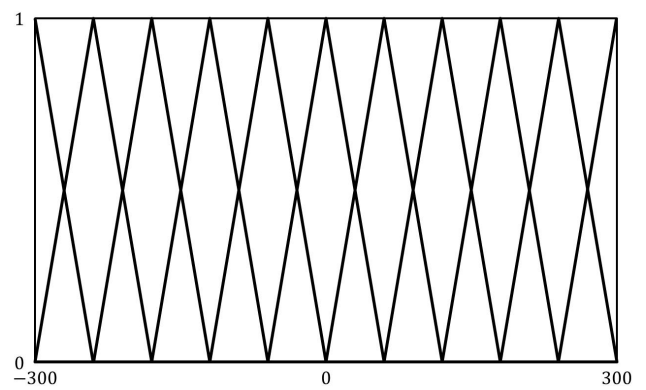

Figure 8. Membership functions for $v$.

ures 12 and 13 . The simulation results show that the proposed model approximates the Duhem model within a bounded range of $\left[\begin{array}{ll}-1.2 & 1\end{array}\right]$. Considering the scaling constant $k=40$, our proposed model has a modeling error within $3 \%$. We conclude that this bounded range is acceptable.

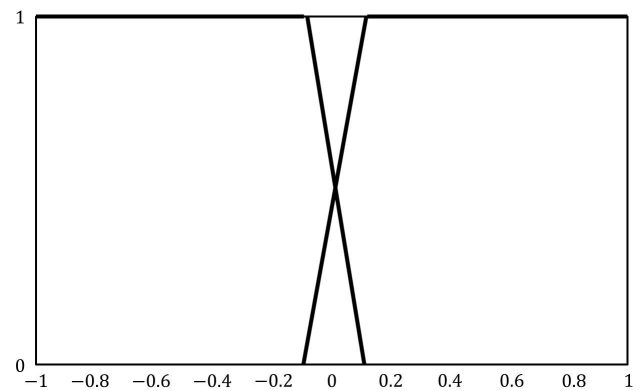

Figure 9. Membership functions for $\dot{v}$.

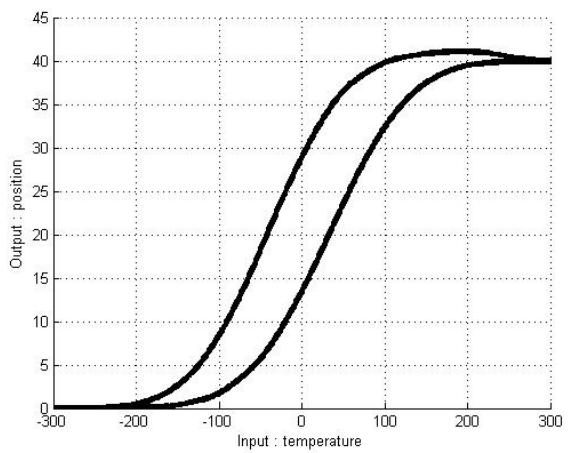

Figure 10. Hysteresis curves for the T-S fuzzy Duhem model with major loop.

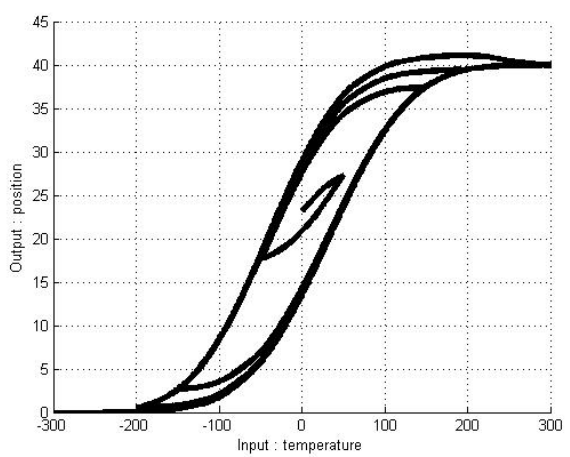

Figure 11. Hysteresis curves for the T-S fuzzy Duhem model with mixed loop.

\section{Conclusion}

Many hysteresis models have been proposed and studied. However, the existing hysteresis models include nonlinear functions. Hence, it is impossible to apply various linear system theories and difficult to analyze a hysteretic system. To solve this problem, we propose a system based on a T-S fuzzy model. To design the T-S fuzzy system, the Duhem model, which is expressed by dynamic equations, can be used. Unlike the previous model, the proposed model can express the local dynamics 


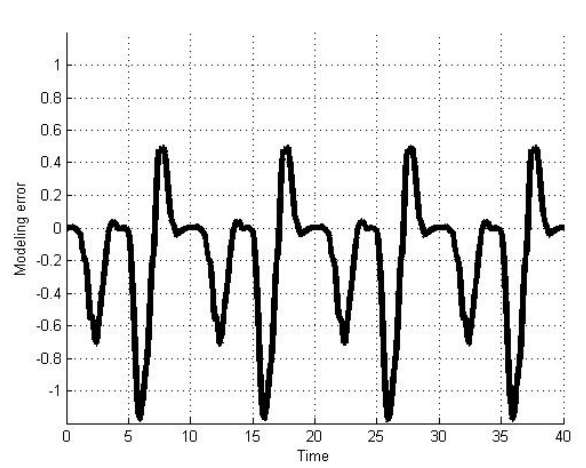

Figure 12. Modeling error for major loop case.

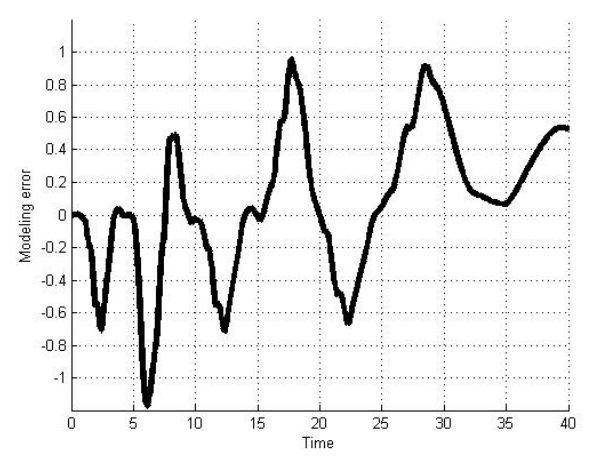

Figure 13. Modeling error for mixed loop case.

of hysteresis with a linear system. Additionally, the proposed model was verified to have applicability to simulations. Considering the wide range of hysteresis modeling, the proposed model is useful.

\section{Conflict of Interest}

No potential conflict of interest relevant to this article was reported.

\section{References}

[1] S. M. Dutta, F. H. Ghorbel, and J. B. Dabney, "Modeling and control of a shape memory alloy actuator," in Proceedings of the 20th IEEE International Symposium on Intelligent Control, Limassol, Cyprus, June 27-29, 2005, pp. 1007-1012. http://dx.doi.org/10.1109/.2005.1467151

[2] I. D. Mayergoyz, "The classical preisach model of hysteresis," in Mathematical Models of Hysteresis, I. D. Mayergoyz, Ed. New York, NY: Springer-Verlag, 1991, pp. 1-63. http://dx.doi.org/10.1007/978-1-4612-3028-1_1
[3] I. Mayergoyz and G. Friedman, "Generalized preisach model of hysteresis," IEEE Transactions on Magnetics, vol. 24, no. 1, pp. 212-217, 1988.

[4] J. W. Macki, P. Nistri, and P. Zecca, "Mathematical models for hysteresis," SIAM Review, vol. 35, no. 1, pp. 94-123, Mar. 1993. http://dx.doi.org/10.1137/1035005

[5] S. M. Dutta, "Dynamic hysteresis modeling and applications," M.S. thesis, Rice University, Houston, TX, 2004.

[6] R. Bouc, "Forced vibration of mechanical systems with hysteresis," in Preceedings of the 4th International Conference on Nonlinear Oscillations, Prague, Czechoslovakia, 1967.

[7] Y. K. Wen, "Method for random vibration of hysteretic systems," ASCE Journal of the Engineering Mechanics Division, vol. 102, no. 2, pp. 249-263, Apr. 1976.

[8] B. D. Coleman and M. L. Hodgdon, "On a class of constitutive relations for ferromagnetic hysteresis," Archive for Rational Mechanics and Analysis, vol. 99, no. 4, pp. 375396, Dec. 1987. http://dx.doi.org/10.1007/BF00282052

[9] L. O. Chua and K. A. Stromsmoe, "Mathematical model for dynamic hysteresis loops," International Journal of Engineering Science, vol. 9, no. 5, pp. 435-450, May 1971. http://dx.doi.org/10.1016/0020-7225(71)90046-2

[10] K. Tanaka and H. O. Wang, Fuzzy Control Systems Design and Analysis: a Linear Matrix Inequality Approach, New York, NY: Wiley, 2001.

[11] L. X. Wang, A Course in Fuzzy Systems and Control, Upper Saddle River, NJ: Prentice Hall PTR, 1997.

[12] S. W. Jun, D. W. Kim, and H. J. Lee, "Design of T-S fuzzy-model-based controller for control of autonomous underwater vehicles," Journal of Korean Institute of Intelligent Systems, vol. 21, no. 3, pp. 302-306, Jun. 2011. http://dx.doi.org/10.5391/JKIIS.2011.21.3.302

[13] H. J. Kim, Y. H. Joo, and J. B. Park, “Controller design for continuous-time Takagi-Sugeno fuzzy systems with fuzzy lyapunov functions: LMI approach," International Journal of Fuzzy Logic and Intelligent Systems, vol. 12, no. 3, pp. 187-192, Sep. 2012. http://dx.doi.org/10.5391/ IJFIS.2012.12.3.187 
[14] M. K. Song, J. B. Park, J. K. Kim, and Y. H. Joo, "Delayrange-dependent stability analysis and stabilization for nonlinear systems: T-S fuzzy model approach," Journal of Korean Institute of Intelligent Systems, vol. 19, no. 3, pp. 337-342, Jun. 2009. http://dx.doi.org/10.5391/JKIIS. 2009.19.3.337

[15] H. J. Lee, Y. H. Joo, S. Y. Lee, and J. B. Park, "Stochastic stabilization of TS fuzzy system with Markovian input delay," Journal of Korean Institute of Intelligent Systems, vol. 11, no. 6, pp. 459-464, Dec. 2001.

[16] Y. Feng, C. A. Rabbath, and C. Y. Su, "Inverse Duhem model based robust adaptive control for flap positioning system with SMA actuators," in Proceedings of the 18th IFAC World Congress, Milano, Italy, August 28September 2, 2011, pp. 8126-8131. http://dx.doi.org/10. 3182/20110828-6-IT-1002.01744

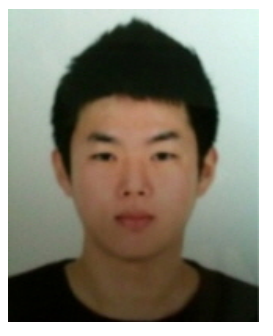

Sang-Yun Lee received his B.S degree at the Department of Electrical and Electronic Engineering in Yonsei University, Seoul, Korea in 2011. He currently takes the M.S. and Ph.D. combined course in the Department of Electrical and Electronic Engineering in Yonsei University, Seoul, Korea, since 2011. His current research interests include intelligent control, nonlinear control, robotics, mobile robots.

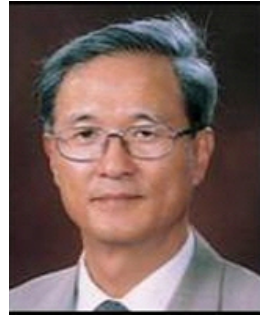

Mignon Park received the B.S. and M.S. in electronics from Yonsei University, Seoul, Korea, in 1973 and 1977, and the Ph.D. from the University of Tokyo, Tokyo, Japan, in 1982. He was a researcher in the Institute of Biomedical Engineering, University of Tokyo, from 1972 to 1982, as well as at the Massachusetts Institute of Technology, Cambridge, and the University of California Berkeley, in 1982. He was a visiting researcher in the Robotics Division, Mechanical Engineering Laboratory Ministry of International Trade and Industry, Tsukuba, Japan, from 1986 to 1987. He has been a Professor in the Department of Electrical and Electronic Engineering, Yonsei University, since 1982. His research interests include fuzzy control and applications, robotics, and fuzzy biomedical systems.

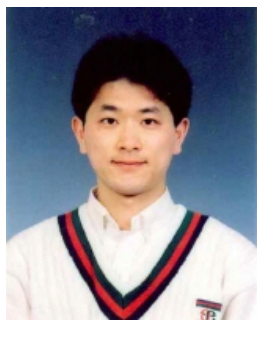

Jaeho Baek received his B.S degree in the Department of Control and Instrumentation Enginearing from Daegu University, Korea,2004. He received his M.S degree and $\mathrm{Ph} . \mathrm{D}$ degree at the Department of Electrical and Electronics engineering in Yonsei university, Korea, in 2006 and 2010

He has been a senior engineer at rhe Samsung, Korea, since 2010. His interests include nonlinear, fuzzy control, chaotic system, robot control, neural networks control. 